\title{
Multivariable Q-parametrization for Rejection of Harmonic Disturbances
}

\author{
Dehri Khadija \\ National Engineering School of \\ Gabes \\ University of Gabes \\ Street of Medenine 6029 \\ Gabes, Tunisia \\ Research Unit: Numerical \\ Control of Industrial Processes
}

\author{
Ltaief Majda \\ National Engineering School of \\ Gabes \\ University of Gabes \\ Street of Medenine 6029 \\ Gabes, Tunisia \\ Research Unit: Numerical \\ Control of Industrial Processes
}

\author{
Nouri Ahmed Said \\ National Engineering School of \\ Gabes \\ University of Gabes \\ Street of Medenine 6029 \\ Gabes, Tunisia \\ Research Unit: Numerical \\ Control of Industrial Processes
}

\begin{abstract}
The design of accurate control becomes more difficult due to the complexity of industrial processes. In this paper, we are interested on the tracking of reference trajectories and the rejection of harmonic disturbances for discrete multi-input multi-output systems.

A new control strategy based on the combination of partial state reference model control, internal model principle and Youla-Kucera parametrization for discrete multivariable systems isproposed. A numerical example shows that the proposed strategy gives a good performance in terms of rejecting harmonic disturbances and reference trajectories tracking.
\end{abstract}

\section{Keywords}

Multivariable control systems, Rejection of harmonic disturbances, Partial state reference model control, Internal model principle, Youla-Kucera parametrization.

\section{INTRODUCTION}

The rejection of disturbances is a problem frequently encountered in control engineering. The disturbances are, often, harmonic and can appears in a variety of industrial processes such as optical and magnetic disk drives, active noise control, rotating machine tools, and robots, etc.

In the literature, there are several main approaches dealing with harmonic disturbances rejection. The most common approach is based on the internal model principle (IMP) [1]. This approach states that the controller should incorporate the model of the disturbance to be eliminated. Other methods are: the adaptive feedforward cancelation (AFC) [2], [3], [4], [5], repetitive control (RC) [6], [7], [8], [9] and iterative learning control (ILC) [10], [11], [12]. However, these strategies (IMC, AFC, RC and ILC) are confronted with the problem of stability. One solution of this problem is to use internal model principle with Youla-Kucera parametrization. Landau et al. [13], [14], [15], [16] present a direct adaptive control based on the internal model principle and the use of the Youla-Kucera parametrization (Q-parametrization) for a single input/singleoutput (SISO) systems. The main idea behind this approach is to insert and adjust the internal model of the disturbance in the controller by tuning the parameters of the polynomial Q.

In practice, many of industrial processes, suffering of harmonic disturbances, are multi-input/multi-output (MIMO) systems (mechanical and electrical systems, helicopter, robotic system, etc). The interactions problem between inputs and outputs, the nonlinearity of the dynamics, the uncertainty of the parameters and/or the presence of external disturbances make the design of a robust control, for MIMO systems, very difficult [17].

This paper focuses on the rejection of harmonic disturbances for discrete MIMO systems. The approach consists in the synthesis of a partial state reference model control (PSRMC) combined with an internal model principle and a YoulaKucera parametrization. Firstly, we develop the partial state reference model control. Then, we adjust the parameters of the controllers using the Q-parametrization. To obtain the elements of polynomial matrix $\mathrm{Q}$, we use a diophantine polynomial matrix in which the model of disturbance is included.

This paper is organized as follows. In section 2, a brief review of Q-parametrization for SISO systems is presented. The third section describes the method for rejection of harmonic disturbances based on the partial state reference model control, internal model principle and Youla-Kucera parametrization. In section 4, the performance of the proposed method is illustrated through a simulation example. Then, we finish by a conclusion.

\section{THE Q-PARAMETRIZATION FOR SINGLE-INPUT SINGLE-OUTPUT SYSTEMS}

Consider a single-input single-output (SISO) discrete-time system described by the following model:

$$
y(k)=q^{-d-1} \frac{B\left(q^{-1}\right)}{A\left(q^{-1}\right)} u(k)+\frac{1}{A\left(q^{-1}\right)} p(k)
$$

where:

$$
\left\{\begin{array}{l}
A\left(q^{-1}\right)=1+a_{1} q^{-1}+a_{2} q^{-2}+\cdots+a_{n_{A}} q^{-n_{A}} \\
B\left(q^{-1}\right)=b_{0}+b_{1} q^{-1}+b_{2} q^{-2}+\cdots+b_{n_{B}} q^{-n_{B}}
\end{array}\right.
$$

$u(k)$ and $y(k)$ are respectively the input and the output of the system.

The model of the system $q^{-d-1} \frac{B\left(q^{-1}\right)}{A\left(q^{-1}\right)}$ is known or obtained by system identification [18].

$p(k)$ is the deterministic external disturbance and can be modeled as: 


$$
p(k)=H\left(q^{-1}\right) \delta(k)
$$

where: $\delta(k)$ is the unit Dirac impulse.

The disturbance transfer function $H\left(q^{-1}\right)$ can be written as:

$$
H\left(q^{-1}\right)=\frac{C\left(q^{-1}\right)}{D\left(q^{-1}\right)}
$$

with:

$$
\left\{\begin{array}{l}
D\left(q^{-1}\right)=1+d_{1} q^{-1}+d_{2} q^{-2}+\cdots+d_{n_{D}} q^{-n_{D}} \\
C\left(q^{-1}\right)=c_{0}+c_{1} q^{-1}+c_{2} q^{-2}+\cdots+c_{n_{C}} q^{-n_{C}}
\end{array}\right.
$$

The controller to be designed is an $R S$-type polynomial which can be expressed as [16]:

$S_{0}\left(q^{-1}\right) \Delta u(k)+R_{0}\left(q^{-1}\right) y(k)=P_{c 0}\left(q^{-1}\right) \beta y_{r}(k+d+1)$

with: $\beta=\frac{1}{n_{B}}, \Delta u(k)=\Delta\left(q^{-1}\right) u(k), \Delta\left(q^{-1}\right)=1-q^{-1}$

$$
\sum_{i=0}^{n_{B}} b_{i}
$$

$y_{r}(k)$ is the desired reference trajectory to be tracked. $S_{0}\left(q^{-1}\right)$ and $R_{0}\left(q^{-1}\right)$ are polynomials characterizing the controller. The desired dynamics of the closed loop system is defined in the the polynomial $P_{c 0}\left(q^{-1}\right)$ as:

$P_{c 0}\left(q^{-1}\right)=\Delta\left(q^{-1}\right) A\left(q^{-1}\right) S_{0}\left(q^{-1}\right)+q^{-d-1} B\left(q^{-1}\right) R_{0}\left(q^{-1}\right)$

Where: $S_{0}\left(q^{-1}\right)$ and $R_{0}\left(q^{-1}\right)$ are the solutions of the diophantine equation given by (3).

The system given by the equation (1) can be described by the behavior of its input-output tracking errors $e_{u}(k)$ and $e_{y}(k)$ given as follow:

$e_{u}(k)=u(k)-A\left(q^{-1}\right) \beta y_{r}(k+d+1)=-\frac{R_{0}\left(q^{-1}\right)}{P_{c 0}\left(q^{-1}\right)} p(k)$

$e_{y}(k)=y(k)-B\left(q^{-1}\right) \beta y_{r}(k)=\frac{S_{0}\left(q^{-1}\right)}{P_{c 0}\left(q^{-1}\right)} p(k)$

Using the "Youla-Kucera" parametrization, the structure of the stabilizing controller $\left(S\left(q^{-1}\right), R\left(q^{-1}\right)\right)$ has the following form [16]:

$S\left(q^{-1}\right) \Delta\left(q^{-1}\right) u(k)+R\left(q^{-1}\right) y(k)=P_{c 0}\left(q^{-1}\right) \beta y_{r}(k+d+1)$

where:

$S\left(q^{-1}\right)=S_{0}\left(q^{-1}\right)-q^{-d-1} Q\left(q^{-1}\right) B\left(q^{-1}\right)$

$R\left(q^{-1}\right)=R_{0}\left(q^{-1}\right)+Q\left(q^{-1}\right) \Delta\left(q^{-1}\right) A\left(q^{-1}\right)$

with $Q\left(q^{-1}\right)$ is the "Youla-Kucera" parameter written as: The controller given by (6), (7) and (8) achieves asymptotic disturbance rejection when the polynomial $S\left(q^{-1}\right)$ has the form:

$$
S\left(q^{-1}\right)=M\left(q^{-1}\right) D\left(q^{-1}\right)
$$

It follows from (7):

$$
S_{0}\left(q^{-1}\right)=M\left(q^{-1}\right) D\left(q^{-1}\right)+q^{-d-1} B\left(q^{-1}\right) Q\left(q^{-1}\right)
$$

Where $M\left(q^{-1}\right)$ and $Q\left(q^{-1}\right)$ are the unique solutions of the diophantine equation (9) with:

$$
\left\{\begin{array}{l}
M\left(q^{-1}\right)=m_{0}+m_{1} q^{-1}+\ldots+m_{n_{M}} q^{-n_{M}} \\
Q\left(q^{-1}\right)=q_{0}+q_{1} q^{-1}+\ldots+q_{n} Q^{-n_{Q}} \\
n_{M}=n_{B}+d ; n_{Q}=\sup \left(n_{D}-1, n_{S_{0}}-1\right)
\end{array}\right.
$$

Then, the stabilizing controller given by the equations (6), (7) and (8) satisfy the following condition:

$$
\lim _{k \rightarrow \infty} e_{y}(k)=0
$$

\section{THE SYNTHESIS OF Q-PARAMETRIZATION FOR MULTI-INPUTS/MULTI-OUTPUTS SYSTEMS}

Consider a class of multi-input multi-output (MIMO) discretetime system described by the following model:

$$
A\left(q^{-1}\right) Y(k)=q^{-d-1} B\left(q^{-1}\right) U(k)+V(k)
$$

where $Y(k), U(k)$ and $V(k)$ are respectively the output, input and disturbances vectors:

$$
\begin{aligned}
Y(k) & =\left[\begin{array}{lll}
y_{1}(k) & \ldots & y_{p}(k)
\end{array}\right]^{T} \\
U(k) & =\left[\begin{array}{lll}
u_{1}(k) & \ldots & u_{p}(k)
\end{array}\right]^{T} \\
V(k) & =\left[\begin{array}{lll}
v_{1}(k) & \ldots & v_{p}(k)
\end{array}\right]^{T}
\end{aligned}
$$

$A\left(q^{-1}\right)$ and $B\left(q^{-1}\right)$ are two polynomial matrices defined as follows:

$$
\left\{\begin{array}{l}
A\left(q^{-1}\right)=I_{p}+A_{1} q^{-1}+A_{2} q^{-2}+\cdots+A_{n_{A}} q^{-n_{A}} \\
\operatorname{dim} A_{\tau_{1}}=(p, p) ; \tau_{1} \in\left[1, n_{A}\right] \\
B\left(q^{-1}\right)=B_{0}+B_{1} q^{-1}+B_{2} q^{-2}+\cdots+B_{n_{B}} q^{-n_{B}} \\
\operatorname{dim} B_{\tau_{2}}=(p, p) ; \tau_{2} \in\left[0, n_{B}\right]
\end{array}\right.
$$

The disturbances vector $V(k)$ is supposed to be deterministic and it can be considered as the result of a Dirac impulse passed through a specific model filter. It can be modeled as:

$$
V(k)=H\left(q^{-1}\right) \delta(k)
$$

where $\delta(k)$ is the unit Dirac impulse vector.

The transfer matrix $H\left(q^{-1}\right)$ can be written as:

where:

$\left\{\begin{array}{l}D\left(q^{-1}\right)=I_{p}+D_{1} q^{-1}+D_{2} q^{-2}+\cdots+D_{n_{D}} q^{-n_{D}} ; \\ \operatorname{dim} D_{\tau_{3}}=(p, p) ; \tau_{3} \in\left[1, n_{D}\right] \\ C\left(q^{-1}\right)=C_{0}+C_{1} q^{-1}+C_{2} q^{-2}+\cdots+C_{n_{C}} q^{-n_{C}} ; \\ \operatorname{dim} C_{\tau_{4}}=(p, p) ; \tau_{4} \in\left[0, n_{C}\right]\end{array}\right.$

We note $\left(A_{r}\left(q^{-1}\right), B_{r}\left(q^{-1}\right)\right)$ the right factorization of the system model $\left(A\left(q^{-1}\right), B\left(q^{-1}\right)\right)$.

$\left(A\left(q^{-1}\right)\right)^{-1} B\left(q^{-1}\right)=B_{r}\left(q^{-1}\right)\left(A_{r}\left(q^{-1}\right)\right)^{-1}$ 
where:

$$
\left\{\begin{array}{l}
A_{r}\left(q^{-1}\right)=I_{p}+A_{r_{1}} q^{-1}+A_{r_{2}} q^{-2}+\cdots+A_{r_{n_{A}}} q^{-n_{A r}} ; \\
\operatorname{dim} A_{\tau_{5}}=(p, p) ; \tau_{5} \in\left[r_{1}, r_{n_{A_{r}}}\right] \\
B_{r}\left(q^{-1}\right)=B_{r_{0}}+B_{r_{1}} q^{-1}+B_{r_{2}} q^{-2}+\cdots+B_{r_{n_{B}}} q^{-n_{B_{r}}} \\
\operatorname{dim} B_{\tau_{6}}=(p, p) ; \tau_{6} \in\left[r_{0}, r_{n_{B_{r}}}\right]
\end{array}\right.
$$

The input-output tracking errors $E_{U}(k)$ and $E_{Y}(k)$ of the multivariable system can be given as:

$$
\begin{aligned}
& E_{U}(k)=U(k)-A_{r}\left(q^{-1}\right) \beta Y_{r}(k+1+d) \\
& E_{Y}(k)=Y(k)-B_{r}\left(q^{-1}\right) \beta Y_{r}(k)
\end{aligned}
$$

The controller to be designed is a partial state reference model control (PSRMC) [17].

Referring to this control structure we can write:

$S_{0}\left(q^{-1}\right) \Delta U(k)+R_{0}\left(q^{-1}\right) Y(k)=P_{c 0}\left(q^{-1}\right) \beta Y_{r}(k+1+d)$

with: $\quad \beta=\left[B_{r}(1)\right]^{-1}$

$$
\Delta U(k)=\Delta\left(q^{-1}\right) U(k) ; \Delta\left(q^{-1}\right)=\left(1-q^{-1}\right) I_{p}
$$

and $Y_{r}(k)$ is the desired trajectories vector to be tracked.

$S_{0}\left(q^{-1}\right)$ and $R_{0}\left(q^{-1}\right)$ are two polynomial matrices characterizing the controller.

The structure of the (PSRMC) for mimo systems is given in Fig 1.

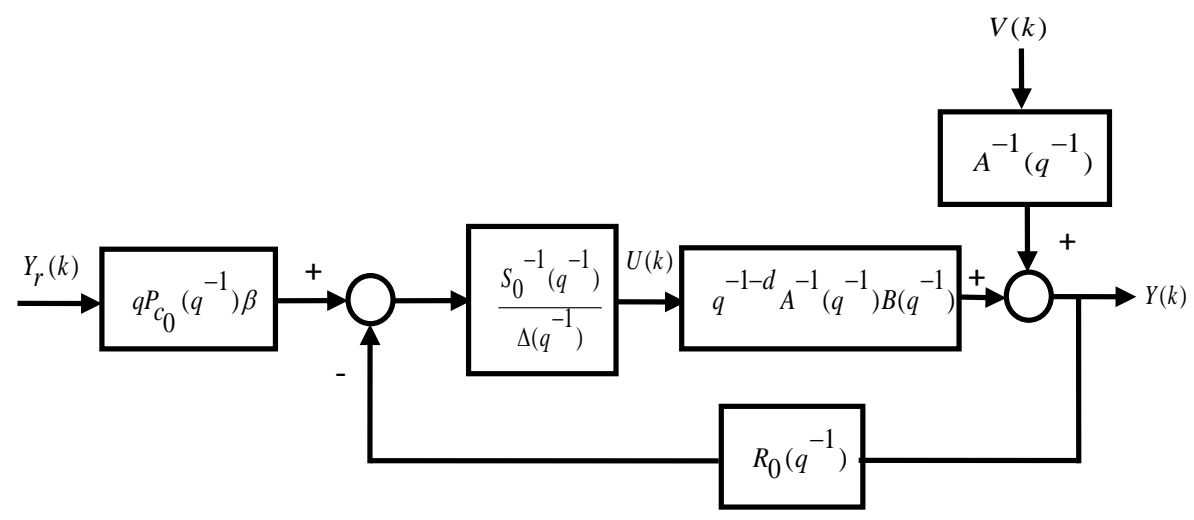

Fig 1: Structure of partial state reference model control.

Let $P_{c 0}\left(q^{-1}\right)$ the polynomial matrix defining the desired dynamics in closed loop:

$$
P_{c 0}\left(q^{-1}\right)=S_{0}\left(q^{-1}\right) \Delta\left(q^{-1}\right) A_{r}\left(q^{-1}\right)+q^{-1-d} R_{0}\left(q^{-1}\right) B_{r}\left(q^{-1}\right)
$$

Where: $S_{0}\left(q^{-1}\right)$ and $R_{0}\left(q^{-1}\right)$ are the particular solutions of the matrix polynomial diophantine equation given by (17).

The right factorization of the nominal controller $\left(S_{0}\left(q^{-1}\right), R_{0}\left(q^{-1}\right)\right)$ is:

$$
\left(S_{0}\left(q^{-1}\right)\right)^{-1} R_{0}\left(q^{-1}\right)=\tilde{R}_{0}\left(q^{-1}\right)\left(\tilde{S}_{0}\left(q^{-1}\right)\right)^{-1}
$$

Using (13), (17) and (18), we obtain the following equation:

$$
\begin{aligned}
& {\left[\begin{array}{cc}
S_{0}\left(q^{-1}\right) & -R_{0}\left(q^{-1}\right) \\
q^{-1-d} B\left(q^{-1}\right) & \Delta\left(q^{-1}\right) A\left(q^{-1}\right)
\end{array}\right]\left[\begin{array}{cc}
\Delta\left(q^{-1}\right) A_{r}\left(q^{-1}\right) & \tilde{R}_{0}\left(q^{-1}\right) \\
-q^{-1-d} B_{r}\left(q^{-1}\right) & \tilde{S}_{0}\left(q^{-1}\right)
\end{array}\right]} \\
& =\left[\begin{array}{cc}
P_{c 0}\left(q^{-1}\right) & 0 \\
0 & P_{c 0}\left(q^{-1}\right)
\end{array}\right]
\end{aligned}
$$

In practice, the industrial processes are complex and the mathematical model describes this system does not represent perfectly their dynamic behavior. Then, the outputs are affected by the external disturbances and the modeling error which can destabilize the system. The external disturbances are often harmonic. To reject these disturbances, we use a family of regulators stabilizing via parametrization of YoulaKucera [19], [20], [21], [22]. The structure of the stabilizing controller for mimo systems is given by the following expressions:

$$
\begin{aligned}
& P_{c 0}\left(q^{-1}\right)=S\left(q^{-1}\right) \Delta\left(q^{-1}\right) A_{r}\left(q^{-1}\right)+q^{-1-d} R\left(q^{-1}\right) B_{r}\left(q^{-1}\right) \\
& S\left(q^{-1}\right)=S_{0}\left(q^{-1}\right)-q^{-1-d} Q\left(q^{-1}\right) B\left(q^{-1}\right) \\
& R\left(q^{-1}\right)=R_{0}\left(q^{-1}\right)+Q\left(q^{-1}\right) \Delta\left(q^{-1}\right) A\left(q^{-1}\right)
\end{aligned}
$$


with $Q\left(q^{-1}\right)$ is the "Youla-Kucera" polynomial matrix written as:

$$
Q\left(q^{-1}\right)=Q_{0}+Q_{1} q^{-1}+Q_{2} q^{-2}+\cdots+Q_{n} q^{-n} Q
$$

The structure of the parametrization of the stabilizing controller is illustrated by the Fig 2 .

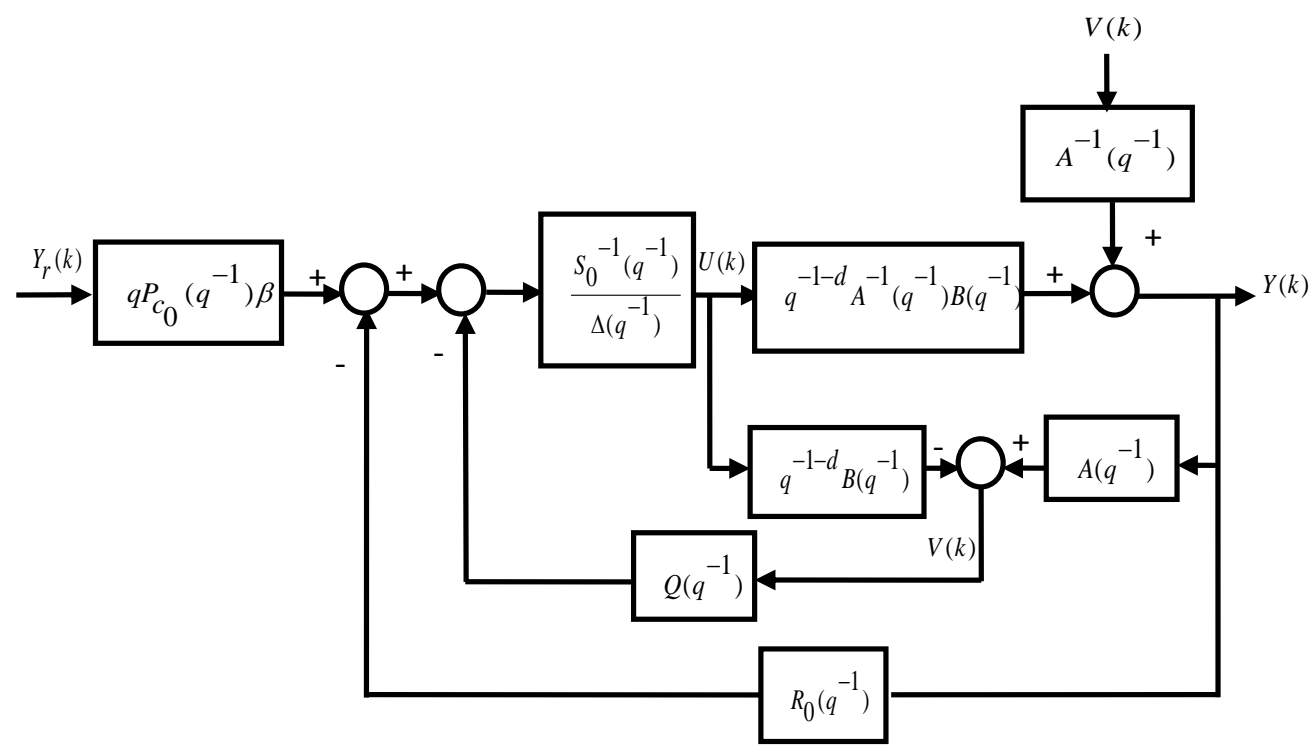

Fig 2: Structure of partial state reference model control combined with the Youla-Kucera parametrization.

The compensation of the disturbance is obtained when the polynomial matrix $S\left(q^{-1}\right)$ is written as:

$$
S\left(q^{-1}\right)=M\left(q^{-1}\right) D\left(q^{-1}\right)
$$

Then the equation (21) can be rewritten as:

$S_{0}\left(q^{-1}\right)=M\left(q^{-1}\right) D\left(q^{-1}\right)+q^{-1-d} Q\left(q^{-1}\right) B\left(q^{-1}\right)$

with:

$$
\left\{\begin{array}{l}
M\left(q^{-1}\right)=M_{0}+M_{1} q^{-1}+M_{2} q^{-2}+\cdots+M_{n_{M}} q^{-n_{M}} \\
Q\left(q^{-1}\right)=Q_{0}+Q_{1} q^{-1}+Q_{2} q^{-2}+\cdots+Q_{n} q^{-{ }^{-n}} Q \\
n_{M}=n_{B}+d ; n_{Q}=\sup \left(n_{D}-1, n_{S_{0}}-1\right)
\end{array}\right.
$$

Where $M\left(q^{-1}\right)$ and $Q\left(q^{-1}\right)$ are the solution of the matrix polynomial diophantine equation (23).

To compensate the external disturbances, we adjust the parameters of the two polynomial matrices $S\left(q^{-1}\right)$ and $R\left(q^{-1}\right)$ using the Youla-Kucera parametrization. The polynomial matrix $Q\left(q^{-1}\right)$ is a result of the solution of a diophantine equation matrix in which the internal model of disturbances $D\left(q^{-1}\right)$ is incorporated.

In this work, we are interested to the problem of harmonic disturbances rejection. The polynomial matrix $D\left(q^{-1}\right)$ which represent the internal model of the disturbances can be calculated as in Table 1.
Table 1. Model of $D\left(q^{-1}\right)$

\begin{tabular}{|c|c|}
\hline Type of harmonic disturbance & \multicolumn{1}{|c|}{$D\left(q^{-1}\right)$} \\
\hline Sinusoidal & $\left(1-2 \cos \left(w T_{e}\right) q^{-1}+q^{-2}\right) I_{p}$ \\
& $w=\frac{2 \pi}{T} ; T=N T_{e}$ \\
\hline Multi-sinusoidal & $\prod_{i=1}^{M}\left(1-2 \cos \left(w_{i} T_{e}\right) q^{-1}+q^{-2}\right) I_{p}$ \\
& $w_{i}=\frac{2 \pi}{T_{i}} ; T_{i}=N_{i} T_{e}, i \in\left[\begin{array}{ll}1 & M\end{array}\right]$ \\
\hline
\end{tabular}

\section{SIMULATION RESULTS}

The purpose of this section, is to study the performance robustness of the proposed controller in the presence of harmonic disturbance rejection.

Consider the mathematical model of an exothermic chemical reactor [23]:

$$
A\left(q^{-1}\right) Y(k)=q^{-1} B\left(q^{-1}\right) U(k)+V(k)
$$

where:

$$
\left\{\begin{array}{l}
A\left(q^{-1}\right)=I_{2}+A_{1} q^{-1}+A_{2} q^{-2} \\
B\left(q^{-1}\right)=B_{0}+B_{1} q^{-1}
\end{array}\right.
$$

with:

$$
A_{1}=\left[\begin{array}{cc}
-1.8630 & 0 \\
0 & -1.8700
\end{array}\right] ; A_{2}=\left[\begin{array}{cc}
0.8669 & 0 \\
0 & 0.8737
\end{array}\right]
$$


$B_{0}=\left[\begin{array}{ll}0.04195 & 0.4758 \\ 0.05824 & 0.1445\end{array}\right] ; B_{1}=\left[\begin{array}{ll}-0.03796 & -0.4559 \\ -0.05403 & -0.1361\end{array}\right]$

$$
\left\{\begin{array}{l}
Y(k)=\left[\begin{array}{l}
y_{1}(k) \\
y_{2}(k)
\end{array}\right] ; \quad Y_{r}(k)=\left[\begin{array}{c}
y_{r 1}(k) \\
y_{r 2}(k)
\end{array}\right] \\
U(k)=\left[\begin{array}{l}
u_{1}(k) \\
u_{2}(k)
\end{array}\right] ; \quad V(k)=\left[\begin{array}{c}
v_{1}(k) \\
v_{2}(k)
\end{array}\right]
\end{array}\right.
$$

We suppose that the disturbances are given by the following model:

$$
V(k)=H\left(q^{-1}\right) \delta(k)=D^{-1}\left(q^{-1}\right) C\left(q^{-1}\right) \delta(k)
$$

where $\delta(k)$ is the unit Dirac impulse vector.

$$
\left\{\begin{array}{l}
D\left(q^{-1}\right)=I_{2}-1.618 I_{2} q^{-1}+I_{2} q^{-2} \\
C\left(q^{-1}\right)=\left[\begin{array}{cc}
0.1469 & 0 \\
0 & 0.1763
\end{array}\right] q^{-1}
\end{array}\right.
$$

The desired trajectories are defined as follows:

$$
Y_{r}(k)=\left[\begin{array}{l}
1 \\
2
\end{array}\right]
$$

In this section, two kinds of controllers are considered: the classical partial state reference model control (PSRMC) and the new partial state reference model control combined with internal model principle and Youla-Kucera parametrization. The evolution of disturbances $v_{1}(k)$ and $v_{2}(k)$ is given in Fig 3.
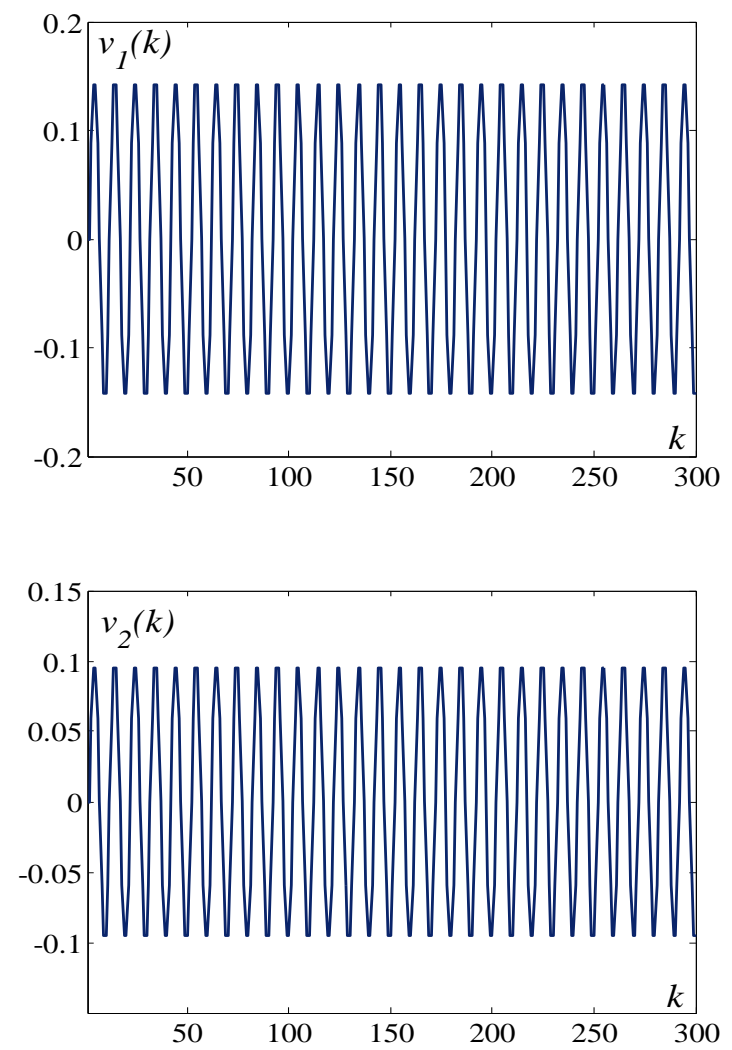

Fig 3: The evolutions of harmonic disturbances $v_{1}(k)$ and $v_{2}(k)$.
Firstly, a classical PSRMC is used. The retained synthesis parameters of the controller are:

$$
\left\{\begin{array}{l}
S_{0}\left(q^{-1}\right)=\left[\begin{array}{ll}
1 & 0 \\
0 & 1
\end{array}\right]+\left[\begin{array}{ll}
-1.0467 & -0.0923 \\
-0.1330 & -2.3567
\end{array}\right] q^{-1} \\
R_{0}\left(q^{-1}\right)=R_{00}+R_{01} q^{-1}+R_{02} q^{-2} ; \\
R_{00}=\left[\begin{array}{cc}
-10.3326 & 35.2136 \\
8.1374 & -2.1408
\end{array}\right] ; R_{01}=\left[\begin{array}{cc}
15.4956 & -53.471 \\
-12.2753 & 3.2499
\end{array}\right] \\
R_{02}=\left[\begin{array}{ll}
-6.1221 & 21.2611 \\
4.8654 & -1.294
\end{array}\right]
\end{array}\right.
$$

The simulation results are shown in Fig 4, Fig 5 and Fig 6.

Fig 4 gives the evolutions of the desired reference trajectories and the outputs of the system. Fig 5 presents the evolutions of the control signals $u_{1}(k)$ and $u_{2}(k)$. The evolution of the output errors $e_{y 1}(k)$ and $e_{y 2}(k)$ is illustrated by the Fig 6. It can be observed that the outputs track the reference trajectories with periodic error due to the presence of external harmonic disturbances. Then, the classical partial state reference model control is not able to reject effectively the considered harmonic disturbances.

Secondly, in order to ameliorate the performance of the classical partial state reference model control, we combine this control with internal model principle and Youla-Kucera parametrization.

The polynomial matrix $Q\left(q^{-1}\right)$ is obtained by solving the diophantine matrix equation (23):

$$
Q\left(q^{-1}\right)=\left[\begin{array}{cc}
-11.322 & 38.6529 \\
8.7521 & -3.2141
\end{array}\right]+\left[\begin{array}{cc}
7.6173 & -27.6518 \\
-10.1313 & 1.326
\end{array}\right] q^{-1}
$$
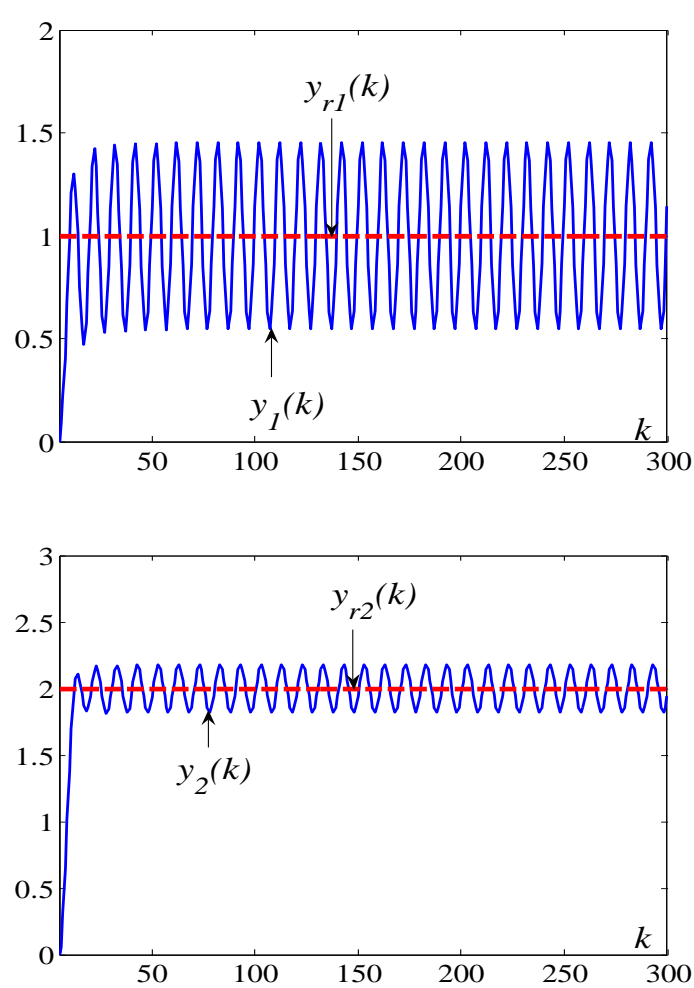

Fig 4: The evolutions of the desired reference trajectories $Y_{r}(k)$ and the output system $Y(k)$ (classical PSRMC). 

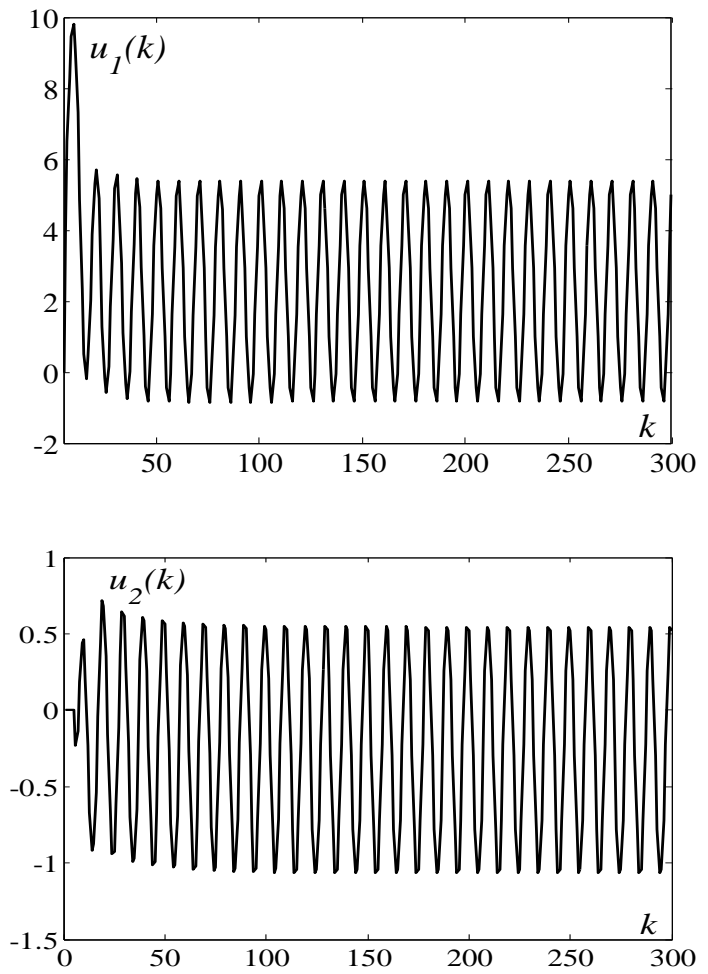

Fig 5: The evolutions of the control inputs $u_{1}(k)$ and $u_{2}(k)$ (classical PSRMC).
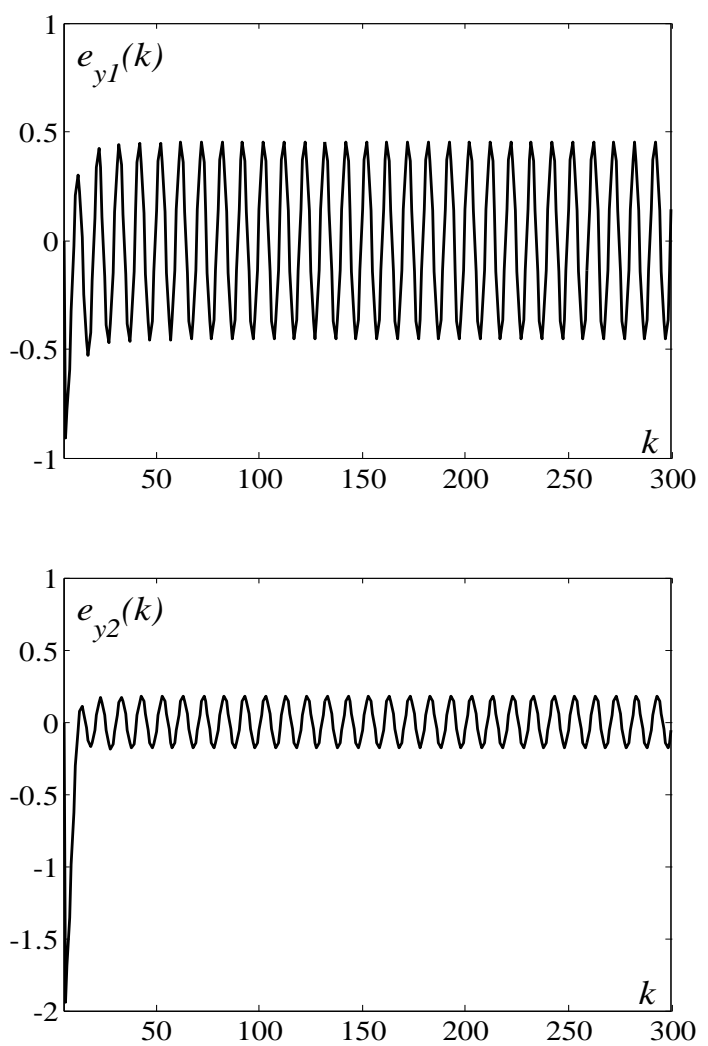

Fig 6: The evolutions of the output errors $e_{y 1}(k)$ and $e_{y 2}(k)$ (classical PSRMC).
After the instant $k>100$, the proposed strategy is applied to the multivariable system. The simulation results of the system with this new strategy are shown in Fig 7, Fig 8 and Fig 9. The evolutions of the desired reference trajectories and the outputs of the system are given in Fig 7. Fig 8 shows the evolutions of the inputs $u_{1}(k)$ and $u_{2}(k)$.

The evolutions of output errors are presented in Fig 9. These figures prove that relatively satisfactory performances are recorded in terms of tracking reference trajectories and rejecting harmonic disturbances. A comparison between the Fig 4 and Fig 7 reveals that the use of the partial state reference model control combined with the Youla-Kucera parametrization reduce effectively the harmonic disturbances.
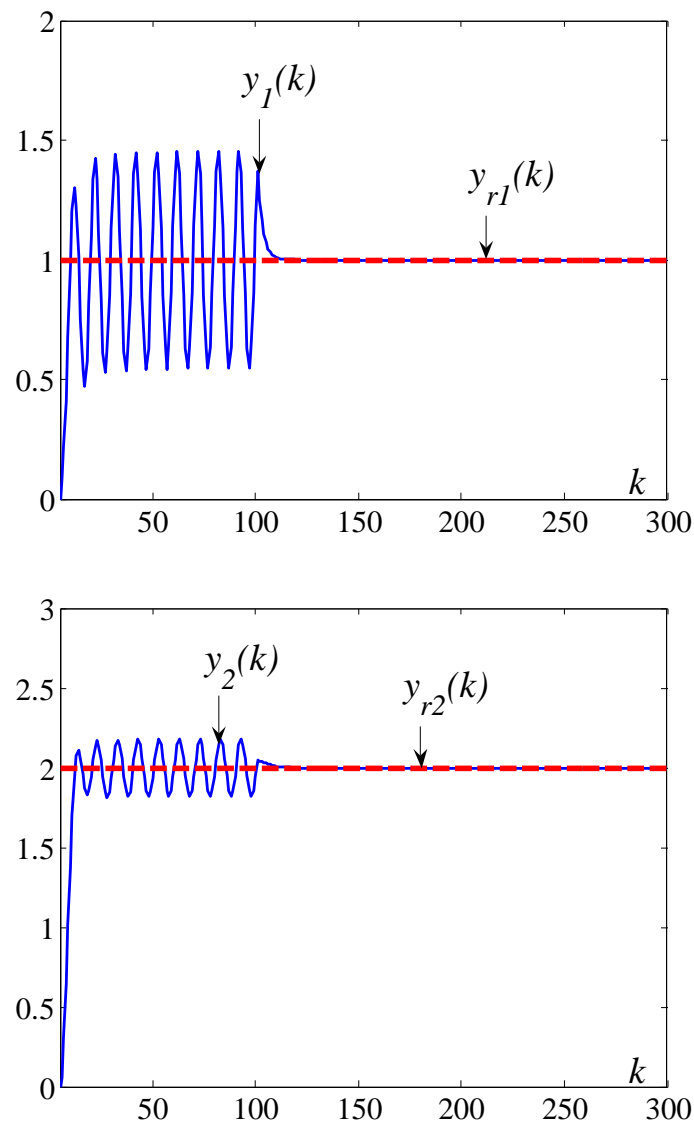

Fig 7: The evolutions of the desired reference trajectories $Y_{r}(k)$ and the output system $Y(k)$ (PSRMC with Qparametrization). 

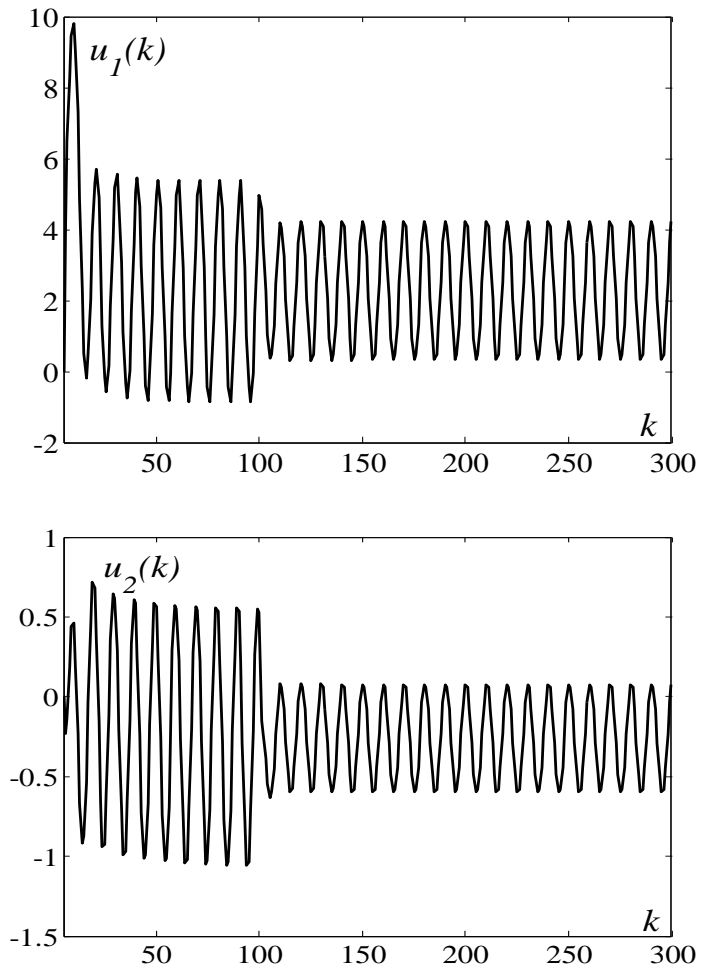

Fig 8: The evolutions of the control inputs $u_{1}(k)$ and $u_{2}(k)$ (PSRMC with Q-parametrization).
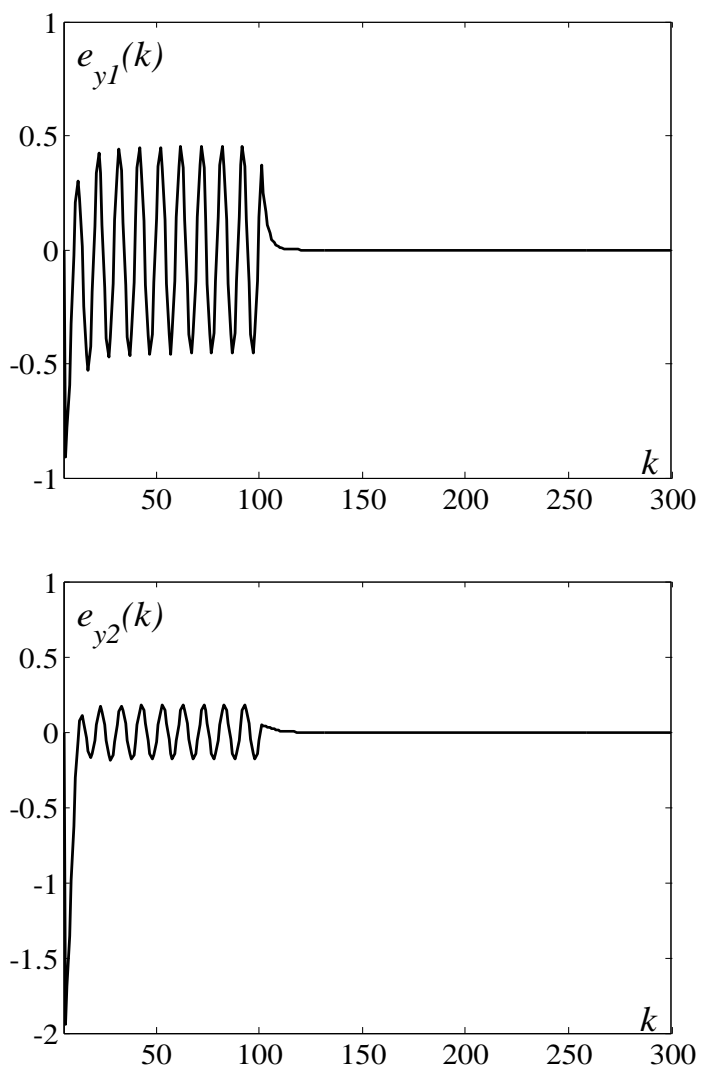

Fig 9: The evolutions of the output errors $e_{y 1}(k)$ and $e_{y 2}(k)$ (PSRMC with Q-parametrization).

\section{CONCLUSION}

In this paper, the problem of rejecting harmonic disturbances for mimo discrete systems was considered. The cancelation of the disturbances is achieved by constructing a set of stabilizing controllers using the Youla-Kucera parametrization. In fact, a new strategy based on the combination of the partial state reference model control, internal model principle and Q-parametrization was proposed. The obtained simulation results show a perfect tracking of reference trajectories and a good rejection of harmonic disturbances for discrete multivariable systems.

\section{ACKNOWLEDGMENTS}

"This work was supported by the Ministry of the Higher Education and Scientific Research in Tunisia”.

\section{REFERENCES}

[1] Francis, B., and Wonham, W. 1976. The internal model principle of control theory. Automatica, vol. 12, no. 5, pp. 457-465.

[2] Bodson, M., and Douglas, S.C., 1997. Adaptive algorithms for the rejection of sinusoidal disturbances with unknown frequency, Automatica, vol. 33, no. 12, pp. 2213-2221.

[3] Bodson, M., 2004. Rejet des perturbations priodiques frquence variable et inconnue, Confrence Internationale Francophone d'Automatique, Douz, Tunisia.

[4] Wu, B., and Bodson, M., 2003. Direct adaptive cancellation of periodic disturbances for multivariable plants, IEEE Transactions on Speech and Audio Processing, vol. 11, no. 6, pp. 538-548.

[5] Wu, B., and Bodson, M., 2004. Multi-channel active noise control for periodic sources-indirect approach, Automatica, vol. 40, no. 2, pp. 203-212.

[6] Arimoto, S., Kawamura, S., and Miyazaki, F., 1984. Bettering operation of dynamic systems by learning: A new control theory for servomechanism or mechatronics systems, Proceedings of 23rd Conference on Decision and Control, pp. 1064-1069.

[7] Hara, S., Yamamoto, Y., Omata, T., and Nakano, M., 1988. Repetitive control system: A new type servo system for periodic exogenous signals, IEEE Transactions on Automatic Control, vol. 33, no. 7, pp. 659-668.

[8] Dehri, K., Ltaief, M., Nouri, A. S. and Ben Abdennour, R., 2011. Rejection of periodic disturbances with unknown frequency for multivariable systems, International Journal on Sciences and Techniques of Automatic control and computer engineering, vol. 5, no. 1, pp. $1458-1471$

[9] Dehri, K., Ltaief, M., Nouri, A. S., 2011. New discrete multivariable sliding mode control for multi-periodic disturbances rejection, 8th International MultiConference on Systems, Signals and Devices, Tunisia.

[10] Chen, Y. Q., Moore, K. L., Yu, J. and Zhang, T., 2006. Iterative learning control and repetitive control in hard disk drive industry - a tutorial, IEEE Trans. on Systems, Man and Cybernetics, Part-C. 
[11] Ryoo, J. R., and Doh, T.- Y., 2008. Feedback based iterative learning control for mimo LTI systems, International Journal of Control Automation and Systems, vol. 6, no. 2, pp. 269-277.

[12] Ahn, H.- S., Chen, Y. Q., and Moore, K. L., 2007. Iterative learning control: brief survey and categorization, IEEE Transactions on Systems, vol. 37, no. 6, pp. 1099-1121.

[13] Alma, M., Landau, I. D., Molina, J. J. M.-, Buche, G., 2010, Suppression adaptative de vibrations en utilisant un actionneur inertiel, Sixime Confrence Internationale Francophone d'Automatique.

[14] Landau, I. D., Alma, M., Martinez, J. J., and Buche, G., 2010, Direct adaptive suppression of multiple unknown vibrations using an inertial actuator, IEEE Conference on Decision and Control.

[15] Landau, I. D., Constantinescu, A., and Alma, M., 2009. Adaptive regulation - Rejection of unknown multiple narrow band disturbances, 17th Mediterranean Conference on Control and Automation.

[16] Landau, I. D., and Patrascu, D., 2005. Adaptive narrow disturbance rejection applied to an active suspension-an internal model principle approach, Automatica, vol. 41, pp. 563-574.
[17] M'Saad, M., and Sanchez, G., 1992. Partial state reference model adaptive control of multivariable systems, Automatica, vol. 28, no. 6, pp. 1189-1197.

[18] Ben Abdennour, R., Borne, P., Ksouri, M., and M'sahli, F., 2001. Identification et commande des procédés industriels, Technip, Paris, France.

[19] Zhanga, W., Allgower, F., and Liu, T., 2006. Controller parameterization for SISO and MIMO plants with time delay, Systems and Control Letters, vol. 55, pp. 794-801.

[20] Horowitz, R., and Mccarmick, J., 1992. A self-tuning control scheme for disk file servos, IEEE Transactions on Magnetics, vol. 27, no. 6, pp. 4490-4495.

[21] Milonidis, E., and Karcanias, N., 2002. Multivariable finite settling time stabilisation: parametrization and properties, IFAC.

[22] Ven den Hof, P. M. J., and de Callafon, R. A., 1996. Multivariable closed loop identification: from indirect to dual-Youla parametrization, Identification Modelling and Control, vol. 9.

[23] Sedraoui, M., 2008. Apport des principales méthodes d'optimisation dans la commande prédictive généralisée multivariable sous contraintes, Thesis. 